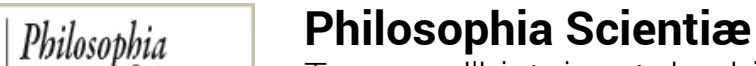

Scientie Travaux d'histoire et de philosophie des sciences

25-2 | 2021

Mathématique et philosophie leibniziennes à la lumière des manuscrits inédits

\title{
Présentation des manuscrits
}

Presentation of the manuscripts

\section{ANR Mathesis}

\section{OpenEdition}

1 Journals

Édition électronique

URL : https://journals.openedition.org/philosophiascientiae/3068

DOI : 10.4000/philosophiascientiae.3068

ISSN : 1775-4283

Éditeur

Éditions Kimé

Édition imprimée

Date de publication : 25 mai 2021

Pagination : 133-154

ISBN : 978-2-38072-015-0

ISSN : 1281-2463

Référence électronique

ANR Mathesis, "Présentation des manuscrits », Philosophia Scientiæ [En ligne], 25-2 | 2021, mis en

ligne le 12 juillet 2021, consulté le 03 novembre 2021. URL : http://journals.openedition.org/

philosophiascientiae/3068; DOI : https://doi.org/10.4000/philosophiascientiae.3068 


\title{
Présentation des manuscrits
}

\author{
ANR « Mathesis»
}

Résumé : Présentation, transcription et traduction de quatre manuscrits mathématiques inédits de Leibniz traitant de la loi de continuité, des logarithmes des nombres imaginaires, des axiomes du calcul des grandeurs et du calcul dyadique.

Abstract: Presentation, transcription and translation of four unpublished mathematical manuscripts by Leibniz dealing with the law of continuity, logarithms of imaginary numbers, axioms for the calculus of magnitudes and the dyadic calculus.

\section{Introduction $^{1}$}

Afin d'indiquer la richesse $\mathrm{du}$ fonds manuscrit de Leibniz, nous présentons ci-dessous quatre courtes pièces inédites avec leurs transcriptions et leur traduction.

Le premier texte fournit un exemple typique et remarquable de dialogue entre philosophie et mathématiques. Il s'agit d'étudier une apparente objection au principe selon lequel on ne doit pas accepter de «Transition par saut » [Regula de Transitu per saltum non admittendo] - une des formulations de la célèbre «loi de continuité » que la tradition a préservée sous la

Philosophia Scientice, 25(2), 2021, 133-154.

1. L'ensemble du dossier a été préparé par : Sandra Bella, Mattia Brancato, Anne Michel-Pajus, Siegmund Probst, David Rabouin et Arilès Remaki à partir de leur travail sur les manuscrits au sein de l'ANR « Mathesis». Comme nous reproduisons les manuscrits, nous n'indiquons pas les corrections et variantes de Leibniz dans les transcriptions et ne reproduisons les diagrammes et les tables que lorsque cela est nécessaire à la lecture de la traduction. 
forme [natura non facit saltus $]^{2}$. L'exception provient ici de la question des puissances de zéro, qui nous confronte à un étrange paradoxe. En faisant varier continûment le paramètre de $0^{x}$ pour $x$ allant des positifs aux négatifs, nous passons brusquement de zéro à un (selon la règle $a^{0}=1$ ) puis à l'infini (puisque $0^{-x}=\frac{1}{0^{x}}=\frac{1}{0}=$ infini). Il s'agit, pourrait-on dire, de concilier un principe de continuité et un principe d'harmonie des formules, qui veut qu'un symbolisme bien construit puisse énoncer la généralité sans exception. La réponse évoquée par Leibniz dès le début du texte est que l'exception n'est qu'apparente parce qu'elle provient du fait qu'ont été introduites des entités « fictives », à savoir le 0 par lequel on divise lorsque l'on explicite l'expression $0^{-x}=\frac{1}{0^{x}}=\frac{1}{0}=$ infini, qui ne saurait être le zéro « absolu » des algébristes (par lequel on ne peut jamais diviser). Ce texte rassemble donc deux des thèmes qui ont été évoqués dans les articles qui précèdent : le problème que pose l'usage du zéro en contexte infinitésimal dès lors qu'il semble violer les règles de l'usage algébrique de ce même symbole ${ }^{3}$; la grande loi caractéristique selon laquelle un symbolisme bien construit doit être à même d'exprimer la généralité qui le sous-tend ${ }^{4}$.

Le diagramme proposé par Leibniz pour «faire voir » cette discontinuité est intéressant. Il figure en effet la ligne des «puissances de zéro » en pointillés, comme courant le long de l'axe des abscisses à une distance qu'il faut donc imaginer comme infiniment petite, puis sautant à 1 en $0^{0}$ et s'échappant ensuite à l'infini (à une distance qu'on imagine infiniment proche du segment unité cette fois). Cette représentation motive le parallèle avec la question des asymptotes à l'hyperbole, qu'il évoque à la fin du texte et dont on pourrait penser qu'elle pose des difficultés comparables (du fait qu'il faudrait imaginer un «saut» pour «atteindre » le point à l'infini $\left.{ }^{5}\right)$. Leibniz répond qu'il n'en est rien dans la mesure où la fiction du point à l'infini figure alors pour une courbe qui doit d'abord passer par tous les segments finis et ne procède donc pas par saut ${ }^{6}$.

La seconde pièce relève également de l'usage des « fictions » et de l'équilibre subtil qui doit s'établir pour Leibniz entre «pensée aveugle » et attestation de possibilité. À partir du moment où nous introduisons des expressions comme 《log » ou « $\sqrt[2]{-1} »$, se pose la question de leur éventuelle combinaison. Puis-je

2. NEEH préface, A VI, 6, 56 : «Rien ne se fait tout d'un coup, et c'est une de mes grandes maximes et des plus verifiées, que la nature ne fait jamais des sauts : ce que j'appellois la loi de la continuité, lorsque j'en parlois autrefois dans les Nouvelles de la République des lettres ; et l'usage de cette loi est trés-considerable dans la physique. » Le texte de 1687 rappelle d'ailleurs que ce principe « a son origine de l'infini, il est absolument necessaire dans la geometrie, mais il reussit encor dans la physique» [GP III, 52].

3. Voir l'article de Sandra Bella ci-dessus p. 21-45.

4. Voir l'article de Simon Gentil ci-dessus p. 47-70.

5. Leibniz est familier de cette approche que nous dirions « projective » depuis ses premières années à Paris, cf. article de Simon Gentil ci-dessus.

6. Sur les usages précoces de la « loi de continuité » dans le maniement des sections coniques, voir l'article de Simon Gentil ci-dessus. 
élever un nombre à la puissance $\sqrt[2]{-1}$ ? Si oui, que vaudrait son logarithme? Le billet explore ces lois de combinaisons symboliques en partant de l'égalité :

$$
\log (a+b \sqrt[2]{-1})+\log (a-b \sqrt[2]{-1})=\log (a a+b b)
$$

On notera au passage la remarquable modernité de ces formules où Leibniz montre, d'une part, qu'il est capable d'écrire l'expression générale de ce que nous appellerions un nombre complexe (tandis que la plupart de ses contemporains voient encore les nombres « imaginaires » comme des solutions particulières, "impossibles », de certaines équations algébriques) et, d'autre part, le rôle que l'on peut faire jouer à la conjugaison (le produit de deux complexes conjugués étant le nombre réel $a a+b b$ ). Il opère ensuite un certain nombre de manipulations formelles pour parvenir à une formule permettant d'exprimer la 《quadrature du cercle » à partir du logarithme. On reste ébloui de tant de prescience, sans équivalent à cette époque, sur la possibilité d'établir des liens entre logarithmes des nombres complexes et fonctions trigonométriques, mais le verso du billet indique les bornes étroites dans lesquelles ces calculs aveugles doivent être tenus : l'expression $x^{\sqrt[2]{-1}}$ reste pour Leibniz une quantité mystérieuse puisque son logarithme $\sqrt[2]{-1} \log (x)$ est une quantité « impossible». Il ajoute alors que le logarithme des nombres négatifs est également une quantité impossible. De fait, on a, selon une ligne d'arguments qu'il mobilise également dans sa querelle avec Jean Bernoulli sur la question, $\log (-x)=2 \log (\sqrt[2]{-x})$. Il qualifie même ce cas de «plus qu'impossible»-ce qui n'est pas sans évoquer un échange avec Wallis où ce dernier qualifie les nombres imaginaires de « doublement impossibles ${ }^{7} »$.

De manière très intéressante, le texte qu'il publie sur ces questions en 1712 dans les Acta eruditorum mobilise également la référence à Jungius sur les énoncés vrais " par tolérance ${ }^{8}$ » et déploie une théorie générale des fictions permettant l'élaboration d'énoncés universels (s'appliquant aussi bien au fait que l'angle de contact est dit par Euclide «plus petit » que tout angle donné, aux grandeurs infiniment grandes et infiniment petites, qu'au rapport de -1 à 1). On y retrouve d'ailleurs les problèmes de continuité de la fonction puissance (le « saut » opérant dans la quadrature des hyperboloïdes entre les puissances positives et négatives, en passant par l'hyperbole ordinaire, ayant conduit Wallis à déclarer les négatifs «plus qu'infinis »).

7. [GM IV, 57]. Wallis considérait les nombres «moins que rien » comme impossibles, leurs racines apparaissant donc à ses yeux comme des impossibles de second degré. Leibniz semble mobiliser un argument similaire pour les logarithmes des imaginaires puisque les logarithmes des négatifs sont déjà impossibles. La situation est néanmoins un peu différente du fait que Leibniz a utilisé le logarithme des imaginaires pour établir l'impossibilité des logarithmes des négatifs, ce qui conduit à une forme de circularité.

8. Observatio, quod rationes sive proportiones non habeant locum circa quantitates nihilo minores, Acta eruditorum, 1712, p. 167-169 [GM V, 387-389]. Voir également la lettre à Wolff, 1713 [GM V, 385]. 
La troisième pièce appartient à un ensemble de textes que Leibniz rédige dans les années 1700 autour de ce qu'il appelle les «fondements du calcul». Il s'agit d'une liste d'axiomes pour le « calcul des grandeurs ». Dans certains de ces textes, Leibniz donne ensuite des démonstrations purement formelles de certaines égalités algébriques ${ }^{9}$. Mais on sera surtout sensible au fait qu'il est donc parvenu à dégager une liste d'axiomes qui évoque celle de ce que nous appelons aujourd'hui un « corps». Cela dit, il convient de ne pas sortir ces textes de leur contexte : cette liste d'axiomes est prise dans un projet plus général dans lequel la structure algébrique n'est pas encore complètement isolée du reste des autres entités mathématiques, y compris celles qui ressortissent du calcul différentiel. Leibniz y revient notamment sur la tension entre manipulation du zéro en contexte algébrique et en contexte infinitésimal. Ainsi un des brouillons préparatoires propose d'établir que « $\frac{a}{0}$ est une absurdité à moins que $a$ ne soit zéro » [LHXXXVIV 12, 7r]. Il ajoute alors un scholie précisant que cet interdit ne s'applique pas au calcul infinitésimal où 0 ne signifie pas un rien pris absolument « mais un nombre qui exprime une grandeur évanouissante ou qui est sur le point de devenir rien, et, qui par conséquent garde encor la nature de la grandeur qu'on suppose décroître continuellement jusqu'à l'évanouissement ${ }^{10} »$.

La quatrième pièce est un des textes sur la dyadique, Periodus numerorum, étudié par Mattia Brancato dans son article ${ }^{11}$. Ce manuscrit est remarquable pour se faire une idée de l'approche « combinatoire » et " caractéristique » de Leibniz, qui dispose sur ces manuscrits de grandes tables où il passe d'un symbolisme à l'autre. Ainsi notre texte introduit-il une nouvelle notation de la forme $0_{2^{j}} 1_{2^{j}}$ pour désigner le nombre « fictif $i j^{12}$. Le nombre $0_{4} 1_{4}$ correspond ainsi au nombre fictif 12 - qui, lui-même, désigne la période de la troisième colonne d'une liste de nombres (en l'occurrence ici, celle de nombres écrits en notation positionnelle binaire et qu'on commence à numéroter à 0 ). Comme nous ne spécifions par s'il s'agit d'un 0 ou d'un 1 , le chiffre 12 correspond ainsi

9. Par exemple : « Si $h=-g$, on aura $-h=g$. En effet, $h=-g$ par hypothèse, donc $g+h=g-g$ (par article 22). Donc $g+h=0$ (par article 25) Donc (par le 23) $g+h-h=0-h$. Donc (par le 22) $g=0-h$, c'est-à-dire (par le 26) $\mathrm{g}=-\mathrm{h}$ » [LH XXXV, 1, 9, fol. 12v] ; trad. fr. [Leibniz 2018, 176, cf. p. 20].

10. [LH XXXV IV 12, fol. 7r], cité ci-dessus p. 41.

11. [LHXXXV, 121 fol. 190-191]. Ce manuscrit n'est pas de la main de Leibniz, mais fut écrit pour Leibniz par Overbeck. Il date vraisemblablement de 1705. Le fol. 190 porte des esquisses de ce qui est développé dans le fol. 191, que nous reproduisons.

12. L'expression «nombres fictifs » ou « feints» n'est pas employée par Leibniz ici, mais dans d'autres textes où il s'en sert pour figurer ce que nous rendons aujourd'hui par des indices. Ils désignent le plus souvent des lignes et des colonnes (désignant, par exemple, les coefficients dans une suite d'équations de même degré). Ici, néanmoins, il s'agit de figurer les nombres dyadiques d'après leur période « selon des colonnes simples ». Le nombre 20 ne désigne donc pas la première colonne de la deuxième ligne, comme dans certains textes sur l'algèbre, mais la colonne qui suit 19 et qui correspond donc à $0_{2} 101_{2} 10$ ou encore $0_{1024} 1_{1024}$. 
à la première colonne d'une expression binaire choisie parmi les 8 combinaisons possibles suivantes, 4 combinaisons commençant par 0 et 4 commençant par 1 :

\begin{tabular}{cccr} 
Expression décimale & \multicolumn{3}{c}{ Expression bin } \\
0 & 0 & 0 & 0 \\
1 & 0 & 0 & 1 \\
2 & 0 & 1 & 0 \\
3 & 0 & 1 & 1 \\
4 & 1 & 0 & 0 \\
5 & 1 & 0 & 1 \\
6 & 1 & 1 & 0 \\
7 & 1 & 1 & 1
\end{tabular}

Lorsqu'on écrit la suite des nombres binaires en partant de 0 , ce tableau correspond aux sept premiers nombres (0 à 7 en décimal) et se reproduit ensuite dans les trois derniers chiffres de tous les nombres qui suivent - d'où l'intérêt de Leibniz pour ces «périodes ». De même, on voit dans cette table que le dernier chiffre d'un nombre binaire est toujours un 0 ou un 1 (période 1 , notée $0_{1} 1_{1}$ ), tandis que l'avant dernier a, pour sa part, une période de $2\left(0_{2} 1_{2}\right)$, etc. Sur la base de la transposition entre ces deux symbolismes, dont il est le créateur, Leibniz entreprend alors de donner des « lois du calcul». On retrouve là à la fois son exceptionnelle inventivité en matière de symbolisme et sa conviction que la recherche des lois structurelles des symbolismes permet, selon un principe d'harmonie, de pénétrer jusqu'au plus profond des choses elles-mêmes ${ }^{13}$.

13. Selon l'expression célèbre de la lettre à Tschirnhaus de 1678 : « nemo autem vereri debet, ne Characterum contemplatio nos a rebus abducat, imò contra ad intima rerum ducet » (fin mai-début juin 1678) [A II, 1, 413]. 


\title{
2 Transcriptions et traductions
}

\author{
$[\mathrm{LH} \mathrm{XXXV,} \mathrm{4,} \mathrm{12,} \mathrm{fol.} 4 \mathrm{r}$ et $4 \mathrm{v}]$ \\ (Regula de transitu per saltum non admittendo ${ }^{14}$ )
}

Regula de Transitu per saltum non admittendo mirabilem quandam exceptionem pati videtur, sed ea oritur ex fictitiis expressionibus, calculo utilibus, attamen ut Jungius loqui solebat, non nisi toleranter veris. Constat $n^{0}$ esse 1, quicunque sit numerus $n$. Itaque etiam locum habebit, cum $n=0$, seu erit $0^{0}=1$. Ita ut $0^{0}$ sit plus quam $0^{1}$ vel $0^{2}$ vel $0^{3}$, etc. Quod ipsum satis paradoxum est, ut exponens minor in rationalibus integris faciat potentiam majorem. Sed jam ad instantiam contra regulam de Saltu non admittendo veniamus. Ita forte non licet adhibere potentias ipsius 0 .

Sit $0^{a-x}$. Hic si $x$ sit $0,1,2,3$, etc. vel alius quivis numerus, modo minor quam $a$, (quem pono esse positivum seu nihilo majorem) erit $0^{a-x}=0$. Sed illo ipso momento quo $x$ pervenit ad $a$, ut fiat $0^{a-a}$ seu $0^{0}$, a 0 transitur ad 1 . Et omnes numeri inter 0 et 1 medii, seu fractiones unitate minores transsiliuntur, quia $0^{0}=1$. Quod si $x$ continuet crescere ultra $a$, statim novus fit saltus adhuc mirabilior, ab unitate ad infinitum, nullo interposito. Sit enim jam $x=a+y$ fiet $a-x=-y$ et $0^{a-x}=0^{-y}=\frac{1}{0^{y}}$. Sed hoc est quantitas infinita, nam si $y$ sit quantitas positiva major nihilo, erit $0^{y}=0$ et $\frac{1}{0^{y}}=\frac{1}{0}=$ infinito. Itaque Nihilum, Unitas et infinitum sese immediate consequuntur, nullo interposito. Et $0^{a-x}$ est 0 , si $x$ sit citra $a$, unitas, si $x$ cadat in $a$; et infinitum si $x$ procedat trans $a$.

14. Transcription et traduction par Anne Michel-Pajus et Sandra Bella, révisées par Siegmund Probst et David Rabouin. 


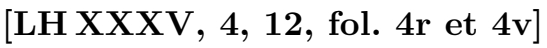

La règle qui consiste à rejeter les Transitions par saut semble souffrir quelque exception admirable, mais celle-ci provient [de l'emploi] d'expressions fictives, utiles pour le calcul, mais cependant, comme Jungius avait coutume de les appeler, vraies seulement par tolérance ${ }^{15}$. C'est un fait établi que, quel que soit le nombre $n, n^{0}$ fait 1 . Cela se produira donc également quand $n=0$, c'est-à-dire qu'on aura $0^{0}=1$. De sorte que $0^{0}$ est plus grand que $0^{1}$ ou $0^{2}$ ou $0^{3}$, etc. Or ceci est déjà assez paradoxal, qu'un exposant plus petit dans les entiers rationnels fasse une puissance plus grande. Mais venons-en dès maintenant à l'exemple contre la règle d'exclusion des Sauts. Dans ce cas, en effet, peut-être n'est-il pas permis de recourir aux puissances du 0 .

Soit $0^{a-x}$. Ici que $x$ soit $0,1,2$, etc. ou un autre nombre au choix plus petit que $a$ (que je pose positif, c'est-à-dire plus grand que rien), on aura $0^{a-x}$. Mais au moment précis où $x$ parvient à $a$, de sorte qu'il en résulte $0^{a-a}$, c'est-àdire $0^{0}$, on passe de 0 à 1 . Et tous les nombres placés comme intermédiaires entre 0 et 1 , c'est-à-dire les fractions plus petites que l'unité, sont transportés d'un bond, parce que $0^{0}=1$. Que si $x$ continue à croître au-delà de $a$, il y aura un nouveau saut subit encore plus admirable, de l'unité à l'infini sans rien entre les deux. Soit en effet maintenant $x=a+y$, on aura $a-x=-y$ et $0^{a-x}=0^{-y}=\frac{1}{0^{y}}$, mais ceci est une quantité infinie, en effet si $y$ est une quantité positive plus grande que rien, on aura $0^{y}=0$ et $\frac{1}{0^{y}}=\frac{1}{0}=$ infini. Ainsi le Rien, l'Unité et l'Infini se suivent immédiatement, sans rien entre eux. Et $0^{a-x}$ est 0 si $x$ est plus petit que $a$, l'unité si $x$ tombe sur $a$ et l'infini, si $x$ va au-delà de $a$.

15. Voir Lettre à Wolff, 1713 [GM V 385]. 
Ut saltum hunc in figura ${ }^{16}$ utcunque exhibeamus. Esto recta $B C$, a puncto $B$ ad $C$, et ultra indefinite producta, in ea sumatur $A B=a$. Sumto A puncto inter $B$ et $C$. Porro sumto quocunque puncto $\xi$ in recta $B C$, sit $B \xi=x$. Jam ducatur linea punctata $F \nu H I K L M$. Ex cujus puncto quocunque ducatur perpendicularis ad rectam $B C$, quales $F B, G \xi, H D, K C, M E^{17}$ et hae vocentur $v$, sitque $0^{a-x}=v$. Patet $F B, \nu \xi, H D$ fore nihilo aequales, seu hactenus lineam punctatam procedere per ipsam $B C$, sed eo momento quo venit ad $A$, longe ab ea prosilire et $K A$ fieri $=a$ posita $a$ representare unitatem, progrediendo autem infra $C$ a $K$ vel $L$ statim prosilire multo ad huc amplius, imo in infinitum ad $M$, ita ut perpendicularis a $M$ ad rectam $B C$ nempe $M E$ sit infinita. Ita tria $x$ sibi indefinite propinqua $H D, 0 ; K A, 1$; et $M E$, infinitum erunt sibi immediata.

Sed qui in quavis Asymptota, qualis Hyperbolae, est saltus, non est Regulae adversus. Ibi enim crescit ordinata ad latitudinem quavis data majorem, antequam infinita fieri intelligatur adeoque per omnes rectas infinita minores. Hic vero subito transitur a 0 ad 1 , non tamen per numeros majores nihilo minores vero unitate. Et similiter statim transitur ab unitate ad infinitum, nec tamen per numeros Unitate majores, et infinito minores. Cujus aliud exemplum non novi.

16. Nous ne transcrivons que le texte. Voir la reproduction du manuscrit ci-dessous p. 150 pour les figures.

17. Le diagramme final (au verso) est précédé de trois diagrammes (au recto), deux inachevés et barrés, puis un troisième (non barré), presque identique au diagramme final sauf pour les notations des points. Dans un premier temps, Leibniz s'appuie sur le diagramme (barré) au recto pour écrire une première version de son texte qu'il corrige ensuite en tenant compte des changements de notations du diagramme final, mais il oublie certaines corrections. Le diagramme représente en pointillés le point courant $\nu$ en fonction du point courant $\xi$. Le G qui apparaît dans le texte est un exemple d'oubli de correction, Leibniz a remplacé les occurrences de $G$ par $\nu$ dans les autres mentions (et $G$ apparaît dans le diagramme recto là où $\nu$ apparaît au diagramme final). Il a également corrigé un $I D$ en $H D$, un $I C$ en $K C$ (le $I$ apparaît deux fois dans le diagramme verso remplacé ensuite par $H$ et $K$ ). Le $C$ sera remplacé par un $A$ (comme indiqué dans la suite du texte). Finalement, le dernier segment porte un $M$ (après hésitations pour la lettre $L$ ). Le point $M$ n'est pas visible sur le diagramme final, mais cela n'est pas surprenant s'il est... à l'infini. Il apparaît cependant timidement dans le diagramme au recto. 
Afin de montrer en quelque manière le saut dans une figure : que soit tirée la droite $B C$ du point $B$ au point $C$, et qu'elle soit prolongée indéfiniment au-delà. On y prend $A B=a$, le point $A$ étant pris entre $B$ et $C$. On prend ensuite un point quelconque $\xi$ sur la droite $B C$, soit $B \xi=x$. Maintenant, que soit menée la ligne en pointillé $F \nu H I K L M$. D'un point quelconque de celle-ci est menée une perpendiculaire à la droite $B C$, comme $F B, G \xi, H D, K C$, $M E$, et celles-ci sont appelées $v$, et que l'on ait $0^{a-x} \cdot=v$. Il est évident que $F B, \nu \xi, H D$ seront égaux à rien, c'est-à-dire que jusque-là la ligne pointillée suit $B C$ elle-même, mais au moment où elle parvient à $A$, elle fera un bond en avant loin d'elle et $K A$ deviendra égal à $a$. En posant que $a$ représente l'unité, en avançant jusqu'à $C$ par en-dessous, elle s'élancera aussitôt à partir de $K$ ou $L$ pour filer beaucoup plus loin, jusqu'à l'infini vers $M$, de sorte que la perpendiculaire menée de $M$ à la droite $B C$, à savoir $M E$, soit infinie. Ainsi trois $x$ indéfiniment proches l'un de l'autre, $H D(0) ; K A(1)$ et $M E$ (infini) seront immédiatement l'un à la suite de l'autre.

Mais le saut qui [apparaît] dans l'une des asymptotes de quelque Hyperbole, ne va pas contre la règle. Là, en effet l'ordonnée croît jusqu'à une longueur plus grande que toute [grandeur] donnée quelconque avant de devenir infinie - et cela s'entend assurément comme : [en passant] par toutes les droites plus petites jusqu'à l'infini. Ici, en revanche, on passe soudainement de 0 à 1 , mais sans passer pour autant par des nombres plus grands que rien et plus petits que l'unité. Et de même une transition s'effectue aussitôt de l'unité à l'infini, mais sans passer pour autant par des nombres plus grands que l'Unité et plus petits que l'infini. Ce dont je n'ai pas connaissance d'autres exemples. 


\section{[LH XXXV, 5, 14, fol. $14 \mathrm{r}$ et 4v]}

\section{(Logarithmus impossibilium $^{18}$ )}

\section{Fol. 14r}

Si log. impossibilium saltem imaginarie exprimi posset calculo foret

$$
\begin{aligned}
& \log . a+b \sqrt{ }-1+\log a-b \sqrt{ }-1=\log a a+b b \\
& \text { et } \log \overline{a a+b b}=\int \frac{d a+d b \sqrt{ }-1}{a+b \sqrt{ }-1}+\int \frac{d a-d b \sqrt{ }-1}{a-b \sqrt{ }-1} \\
& \text { Seu } \left.\frac{d \overline{a a+b b}}{a a+b b}=\begin{array}{l}
a d a+a d b \sqrt{ }-1-b d a \sqrt{ }-1+b d b \\
a d a-a d b \sqrt{ }-1+b d a \sqrt{ }-1+b d b
\end{array}\right\}: a a+b b \\
& \text { fiet } \log \overline{a a+b b}=\int \frac{2 a d a+2 b d b}{a a+b b}
\end{aligned}
$$

Quod consentit.

Itaque dabitur quadratura Circuli per logarithmos, nam si sit $a=y$ inderminata et $b$ sit constans fiet

$$
\log \overline{b b+y y}=2 \int \frac{y d y}{b b+y y}
$$

Hoc accurate experiendum.

\section{Fol. 14v}

Quæritur qualisnam quantitas sit $x \frac{\sqrt[2]{-1}}{2}$ eius logarithmus est $\sqrt[2]{ }-1 . \log . x$. qui est impossibilis.

Impossibilis quantitatis logarithmus est impossibilis [;] impossibilis quantitatis duplus est impossibilis, unde colligitur impossibilem esse logarithmum quantitatis negativæ. Cum duplus sit logarithmi impossibilis, nempe de impossibili ; radice scilicet quadratica quantitatis negativæ. Hinc colligitur logarithmum impossibilis quantitatis esse plus quam impossibilem.

18. Transcription et traduction par Arilès Remaki, révisées par Siegmund Probst et David Rabouin. 


\section{[LH XXXV, 5, 14, fol. 14r et 14v]}

\section{Fol. 14r}

Si l'on pouvait exprimer le log des impossibles, au moins de manière imaginaire, ce serait par ce calcul :

$$
\begin{aligned}
& \log (a+b \sqrt{-1})+\log (a-b \sqrt{-1})=\log \left(a^{2}+b^{2}\right) \\
& \text { et } \log \left(a^{2}+b^{2}\right)=\int \frac{d a+d b \sqrt{-1}}{a+b \sqrt{-1}}+\int \frac{d a-d b \sqrt{-1}}{a-b \sqrt{-1}} \\
& \text { C'est-à-dire } \frac{d\left(a^{2}+b^{2}\right)}{a^{2}+b^{2}}=\frac{+a d a+a d b \sqrt{-1}-b d a \sqrt{-1}+b d b}{a^{2}+b^{2}} \\
& \text { ce qui donne } \log \left(a^{2}+b^{2}\right)=\int \frac{2 a d a+2 b d b}{a^{2}+b^{2}}
\end{aligned}
$$

Ce qui est concordant.

Et c'est ainsi qu'on donnera la quadrature du cercle par les logarithmes, en effet si on a $a=y$ indéterminée et $b$ constante, alors on obtient :

$$
\log \left(b^{2}+y^{2}\right)=2 \int \frac{y d y}{b^{2}+y^{2}}
$$

Il faut tester cela avec minutie.

\section{Fol. 14v}

Soit $x^{\sqrt[2]{-1}}$, dont le logarithme est $\sqrt[2]{-1} \log x$ qui est impossible, on cherche donc de quelle sorte de quantité il s'agit.

Le logarithme d'une quantité impossible est impossible; le double d'une quantité impossible est impossible, on en conclut donc que le logarithme d'une quantité négative est impossible. Puisque c'est le double du logarithme d'un impossible. Et il s'agit bien d'un impossible, à savoir la racine carrée d'une quantité négative. On en conclut à présent que le logarithme d'une quantité impossible est plus qu'impossible. 
[LH XXXV, 4, 12, fol. 2r et 2v]

Elemens du calcul $^{19}$

\section{Axiomes}

(1) chaque grandeur est égale à elle-même

$a=a$

(2) ce qui est egal à un autre peut être mis a sa place dans les estimations des grandeurs

(3) $a+b=b+a$

ainsi $3+2=2+3=5$

(4) $a-a=0$

$1-1=0 \quad 2-2=0 \quad 3-3=0$ et ainsi dans les autres

(5) $0+a=a$

$0+1=1 \quad 0+2=2 \quad 0+3=3$, etc.

(6) $a b=b a$

$3.2=2.3=6$

(7) $0 . a=0$

$0^{\wedge} 1=0 \quad 0^{\wedge} 2=0 \quad 0^{\wedge} 3=0$ etc.

(8) $1 \mathrm{a}=\mathrm{a}$

$1^{\wedge} 1=1 \quad 1^{\wedge} 2=2 \quad 1^{\wedge} 3=3$, etc.

(9) $-a=-1^{\wedge} a$

$-1=-1^{\wedge} 1 \quad-2=-1 \frown 2 \quad-3=-1^{\wedge} 3$

(10) $a, b+c=a b+a c$

$3,1+2=3.1+3.2=3+6=9$

(11) $\frac{a}{a}=1$

$\frac{1}{1}=1 \quad \frac{2}{2}=1 \quad \frac{3}{3}=1$, etc.

(12) $a: \frac{1}{b}=\frac{a}{b}$ ou bien $a: b$

$2 \frac{1}{3}=\frac{2}{3}$

(13) $a^{0}=1$

(14) $a^{1}=a$

(15) $\mathrm{e}(\sqrt[e]{a})=a$

$2 \sqrt[2]{4}$ ou bien le quarré, de la racine quarrée de 4 ; est 4 .

et $3 \sqrt[3]{8}$, ou bien le Cube, de la racine cubique de 8 ; est 8 .

19. Transcription par Anne Michel-Pajus, révisée par Miguel Palomo et David Rabouin. 


\section{Supposition}

On suppose la vérité des valeurs des nombres. Par exemple que $3+2$ est 5 , et que $3 \cdot 2$ est 6 , et choses semblables.

\section{Demande}

On demande qu'il soit permis de prendre pour un nombre tout ce qui entre dans les operations des nombres comme un nombre tant qu'on ne prouve pas qu'il est impossible. Ainsi $2-3$ est pris pour un nombre, quoyqu'il soit moindre de rien, savoir -1 , et ce nombre augmenté par 2, donne 1 . Et $\frac{2}{3}$ est pris pour un nombre, quoiqu'il soit moindre que l'unité, et ce nombre multiplié par 3 donne 2. Et $\sqrt[2]{3}$ est pris pour un nombre quoyqu on ne puisse point l'exprimer par le nombre des parties d'une échelle qui se rapporte à l'unité, c'est- à dire quoyqu'il ne soit point exprimable par la repetition des unités, ou par la repetition de quelcune des parties egales de l'unité. On appelle cela un nombre sourd ou irrationel, on dit aussi que ce nombre n'est point commensurable avec l'unité, ou avec quelque nombre, qui a une mesure commune avec l'unité. Cependant, si ce nombre $\sqrt[2]{3}$ est appelé $a, a^{2}$ (ou $a a$ ) donne 3 . 
[LH XXXV, 12, 1, fol. 191]

(Periodus numerorum ${ }^{20}$ )

\section{LEGES Calculi}

1. Multiplicantur hic non singulae notae

per universas Notas commultiplicantes ut alias

Sed tantum singulae Notae per Singulas correspondentes

Non enim lateraliter hic Numeroque in Numerum ducitur

sed descensive Periodus in Periodum

2. Si Periodus correspondens locum totum alterius non impleat, repetatur toties donec eum impleat.

Nam eadem Periodus repetita, manet tamen Periodus aliqua ejusdem Columnae

3. Multiplicans 0 reponit seipsum, 1 reponit Commultiplicantem Addendum 0 reponit Coaddendum, 1 reponit Anticoaddendum ita ut $(1+1)$ faciat $\dot{0}$ gravidum $\dot{0}$, gravidum addito 1 fit 1 gravidum et hoc addito 1 fit $0 \ddot{~ e t c . ~}$

4. gravidum addit Columnae proxime altiori Unitatem Dupliciter gravidum addit unitatem Columnae altiori una intermissa Tripliciter gravidum addit eodem loco unitatem et praeterea Unitatem loco simili proximae altioris columnae Quadrupliciter gravidum addit Unitatem Columnae altiori duabus intermissis etc.

20. Transcription par Mattia Brancato. Nous ne transcrivons ici que le texte, la table qui le précède étant reproduite ci-dessous après la traduction. 


\section{[LH XXXV, 12, 1, fol. 191] \\ (Periodus numerorum ${ }^{21}$ )}

\section{LOIS du calcul ${ }^{22}$}

1. Ici, on ne multiplie pas les marques simples par toutes les marques commultiplicantes ${ }^{23}$ comme on fait ailleurs Mais seulement les marques simples par les simples correspondantes En effet, on ne calcule pas horizontalement nombre à nombre mais en descendant de période à période ${ }^{24}$.

2. Si la période correspondante ne remplit pas la totalité du lieu de la suivante qu'elle soit répétée autant de fois [qu'il faut] pour la remplir Car une même période répétée, elle reste cependant une certaine période de la même colonne ${ }^{25}$.

3. Multiplier par 0 retourne 0 lui-même, par 1 retourne le commultipliant Ajouter 0 retourne le cofacteur, 1 retourne l'anticofacteur ainsi $(1+1)$ fait $\dot{0}$ avec retenue

$\dot{0}$ avec retenue auquel on ajoute 1 fait 1 avec retenue et si on ajoute 1 à ce dernier on obtient $\ddot{0}$, etc. ${ }^{26}$

4. Toute retenue ajoute une unité à la plus proche colonne supérieure Une double retenue ajoute une unité à la colonne supérieure décalée d'un rang

Une triple retenue ajoute une unité à la même place et ainsi Une unité à la prochaine place semblable de la colonne supérieure Une retenue quadruple ajoute une unité à la colonne supérieure déplacée de deux rangs, etc.

21. Traduction par Mattia Brancato et David Rabouin.

22. Les lois correspondent à la table sur la page de droite du manuscrit (que nous reproduisons p. 154). Elles sont précédées sur la page de gauche du manuscrit de deux tables présentant les développements des carrés selon les deux formes exposées dans l'article de Mattia Brancato, p. 71-94.

23. Leibniz ne fait pas seulement preuve d'inventivité symbolique, mais également terminologique, comme on peut le voir à l'introduction des termes commultiplicans, coaddendum, anticoaddendum.

24. De fait, on calcule sur les chiffres indiqués en petit (qui indiquent la période) séparément des chiffres indiqués en grand ( 0 ou 1 , qui indiquent la structure binaire $\mathrm{du}$ calcul).

25. Ce à quoi Leibniz fait référence ici n'est pas parfaitement clair. Il pourrait s'agir de rappeler que chaque période est considérée comme se prolongeant indéfiniment si bien que lorsqu'une période (disons 4) apparaît comme plus petite qu'une autre (disons 8), il faut en fait « compléter» le premier motif en le répétant deux fois de sorte qu'il soit également de hauteur 8 .

26. Comme expliqué dans la section suivante, la retenue correspond à l'ajout d'une unité sur la colonne suivante : $\dot{0}=10, \ddot{0}=100$. C'est la raison pour laquelle $\dot{0}+1=11$, qui se trouve être 1 avec retenue. 
II

II

$\exists$

$\frac{7}{9} \stackrel{\frac{4}{2}}{\frac{2}{4}}$

\& $\exists \quad \delta$

न

กั ก

$\stackrel{\infty}{\sim} \stackrel{\infty}{\sim}$

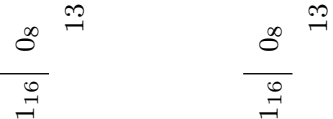

ठ

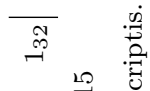

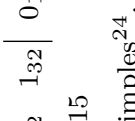

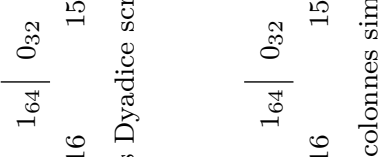

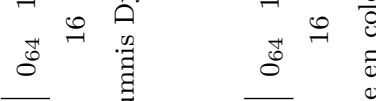

$\begin{array}{lll}\infty & \Xi \\ & 0 & 0 \\ -1 & 0\end{array}$

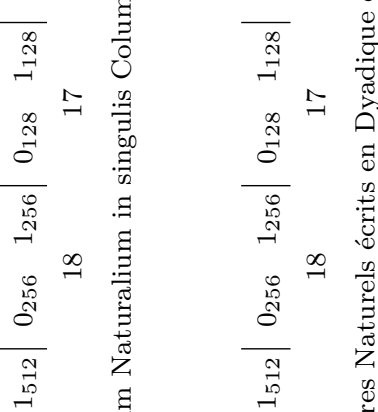

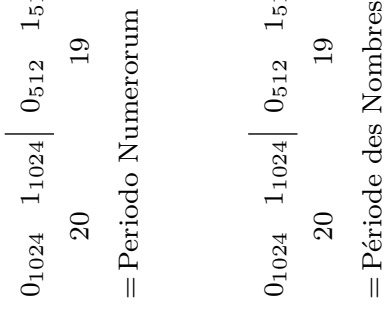




\section{Reproduction des manuscrits}

$\left[\mathrm{LH} \mathrm{XXXV,} \mathrm{4,} \mathrm{12,} \mathrm{fol.} 4 \mathrm{r}\right.$ et $\left.4 \mathrm{v}^{25},\right]$

(Regula de transitu per saltum non admittendo)

Regula de Transitu per Saltum non wirniftekes miratilem quarian exceptionem pati videtur fol

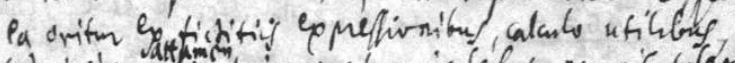

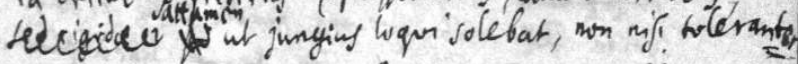

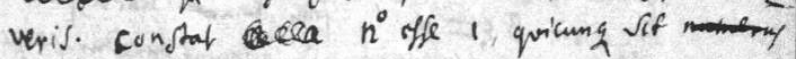
numeny $n$. ifay stiam luarm babrbit com $n=0$, sm mit $0^{0}=1$. ita ut $0^{0}$ sit shy quam $0^{0}$ and $0^{2}$ und $0^{3}$ glete.

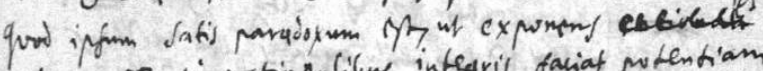
minor in rationaliling integrif faciat putentiam

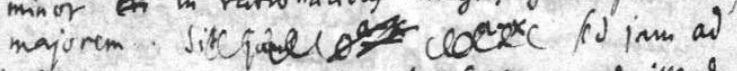
inftantian contra regulam de Saltur non asmittenso

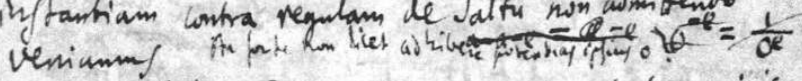
Sib $0^{a-x}$ Hic si $x$ sit $0,1,2,3$, rte unl aldeng quivis

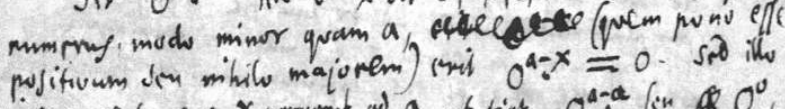
pusitivum den minilo majurem ind $0^{a-X} \equiv 0$. Sed illo

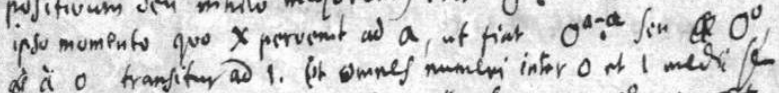

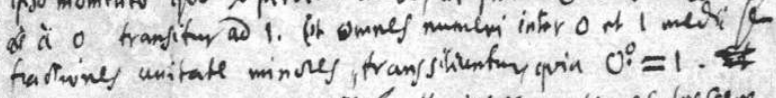

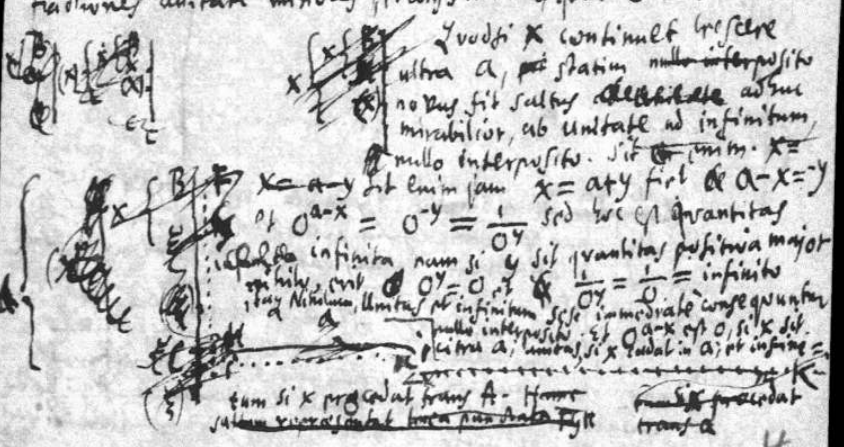

25. Reproduction avec l'aimable autorisation de la Gottfried Willhelm Leibniz Bibliothek - Niedersächsische Landesbibliothek, à Hanovre. https://bit.ly/3fBtX8c, cahier http://digitale-sammlungen.gwlb.de/resolver?id=00068009. 


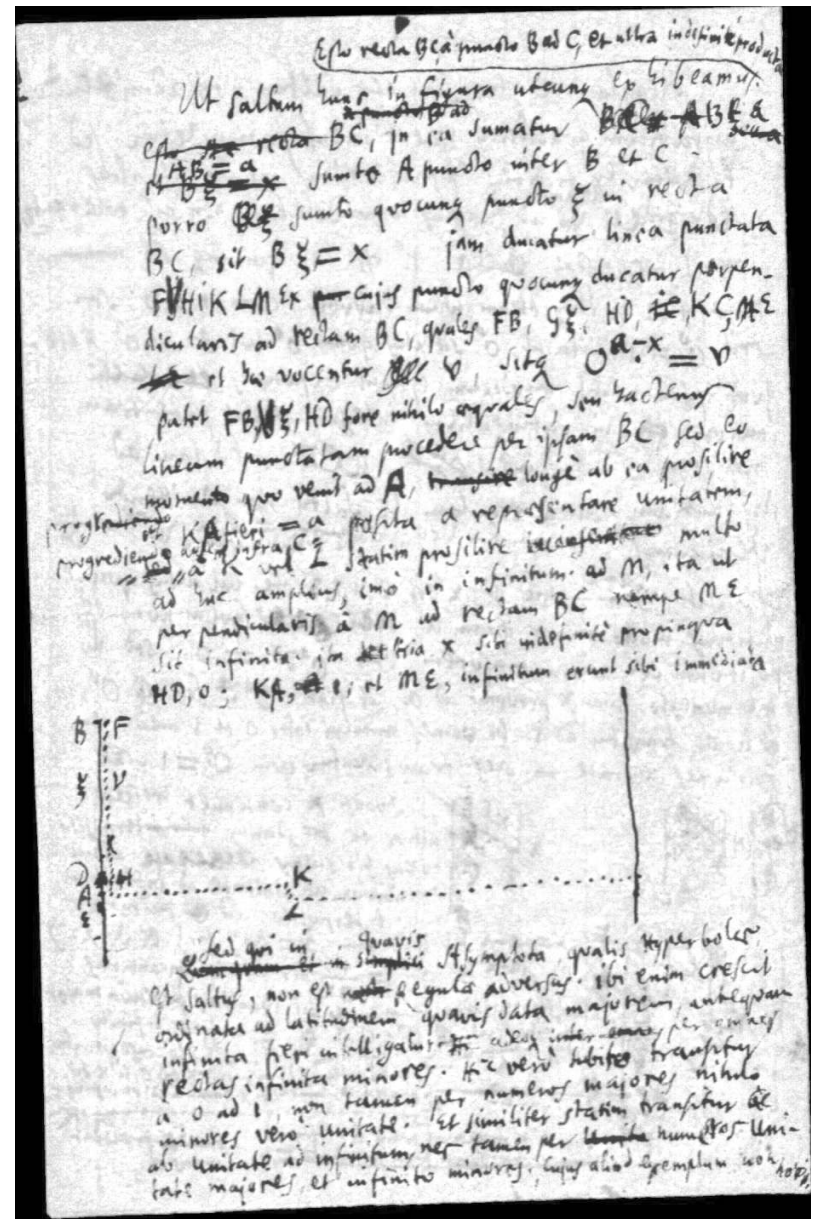


$\left[\mathrm{LH} \mathrm{XXXV,} \mathrm{5,} \mathrm{14,} \mathrm{fol.} 14 \mathrm{r}\right.$ et $\left.14 \mathrm{v}^{26}\right]$

\section{(Logarithmus impossibilium)}
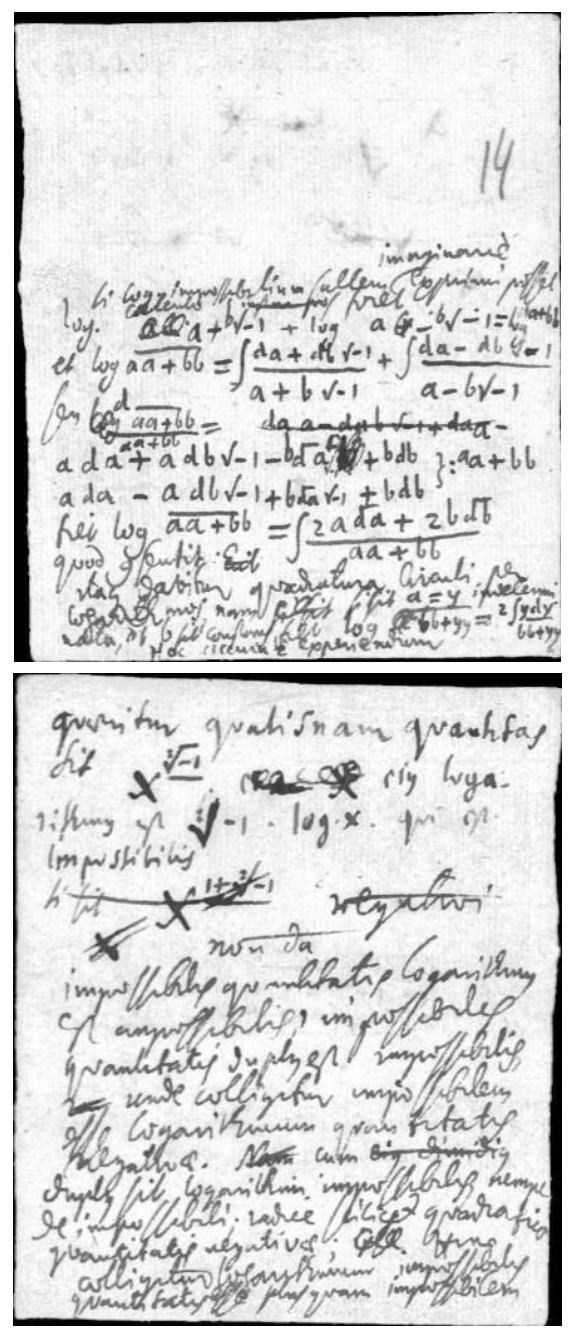

26. Reproduction avec l'aimable autorisation de la Gottfried Willhelm Leibniz Bibliothek - Niedersächsische Landesbibliothek, à Hanovre. https://bit.ly/3ugi8YV ; cahier http://digitale-sammlungen.gwlb.de/resolver?id=00068032. 
[LH XXXV, 4, 12, fol. $2 \mathrm{r}$ et $\left.2 \mathrm{v}^{27}\right]$

(Elemens du calcul)

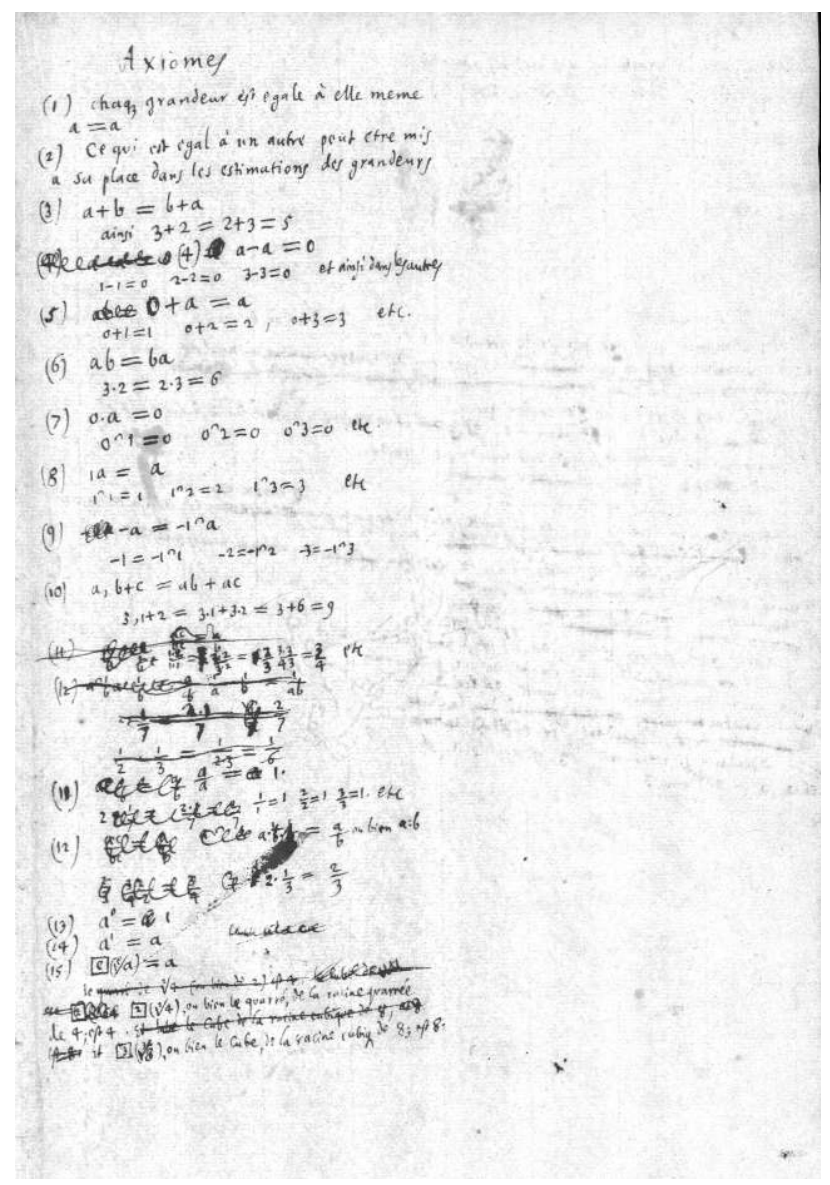

27. Reproduction avec l'aimable autorisation de la Gottfried Willhelm Leibniz Bibliothek - Niedersächsische Landesbibliothek, à Hanovre. https://bit.ly/3wh8FCJ ; cahier http://digitale-sammlungen.gwlb.de/resolver?id=00068009. 


\section{Suppusition}

II On suparse la veritérles valeury def nombreg

lar excomple que $3+2$ is 5 , el que 3.2 ift 6.

it igusesrewblatles

\section{Demande}

On jemande qu'il soit permis de prendive

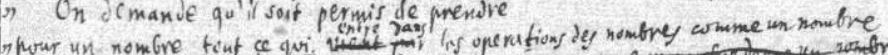

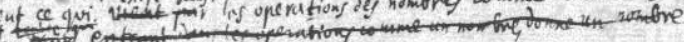

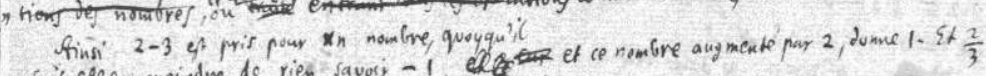
Soit ctecen woindre de rien, savory - 1 , elg limite

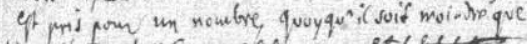

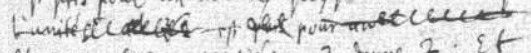

et ce nombre multiplie par 3 donne 2 . Ef

(C) rumbedespinties d'une

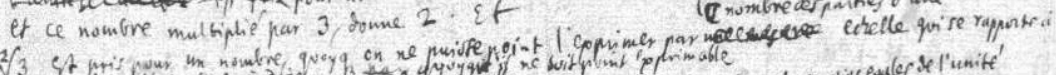

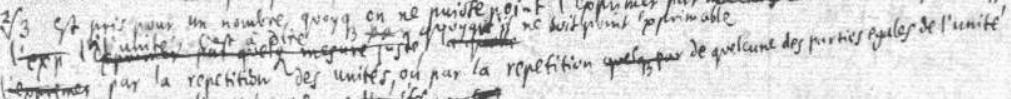

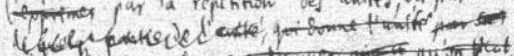

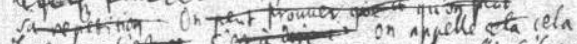

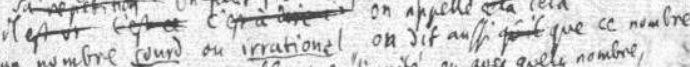

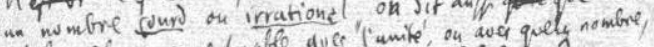

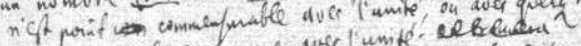

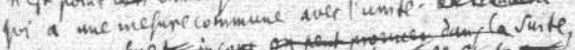

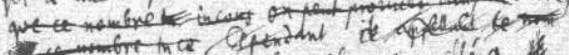

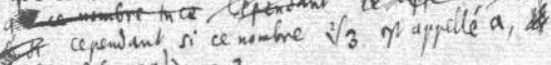
cofes a $a^{2}$ on aa jonne 3 
$\left[\right.$ LH XXXV, 12, 1, fol. $\left.191^{28}\right]$

(Periodus numerorum)

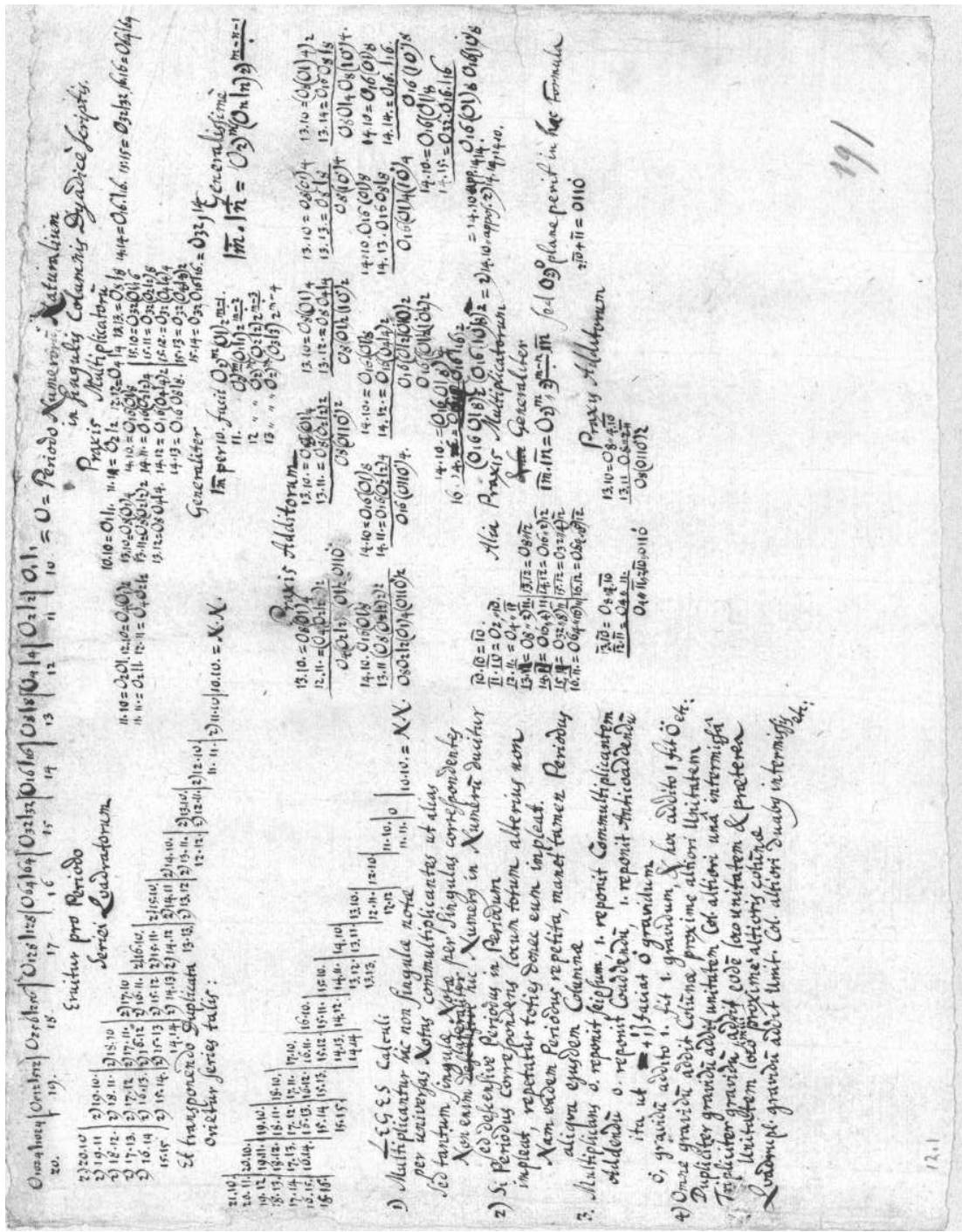

28. Reproduction avec l'aimable autorisation de la Gottfried Willhelm Leibniz Bibliothek - Niedersächsische Landesbibliothek, à Hanovre. https://bit.ly/2Plsd8p ; cahier http://digitale-sammlungen.gwlb.de/resolver?id=00068194. 